\title{
Suicidal Leishmania
}

\author{
Lucie Podešvová ${ }^{1}$, Tereza Leštinová ${ }^{2}$, Eva Horáková ${ }^{3}$, Julius Lukeš ${ }^{3,4}\left(\mathbb{D}\right.$, Petr Volf $^{2}(\mathbb{D}$ and \\ Vyacheslav Yurchenko $1,5, *$ (D) \\ 1 Life Science Research Centre and Institute of Environmental Technologies, Faculty of Science, \\ University of Ostrava, 71000 Ostrava, Czech Republic \\ 2 Department of Parasitology, Faculty of Science, Charles University, 12844 Prague, Czech Republic \\ 3 Biology Centre, Institute of Parasitology, Czech Academy of Sciences, 37005 České Budějovice (Budweis), \\ Czech Republic \\ 4 Faculty of Sciences, University of South Bohemia, 37005 České Budějovice (Budweis), Czech Republic \\ 5 Martsinovsky Institute of Medical Parasitology, Tropical and Vector Borne Diseases, Sechenov University, \\ 119435 Moscow, Russia \\ * Correspondence: vyacheslav.yurchenko@osu.cz; Tel.: +420-597-092-326
}

Received: 8 January 2020; Accepted: 22 January 2020; Published: 25 January 2020

check for updates

\begin{abstract}
Leishmania are obligate intracellular parasites known to have developed successful ways of efficient immunity evasion. Because of this, leishmaniasis, a disease caused by these flagellated protists, is ranked as one of the most serious tropical infections worldwide. Neither prophylactic medication, nor vaccination has been developed thus far, even though the infection has usually led to strong and long-lasting immunity. In this paper, we describe a "suicidal" system established in Leishmania mexicana, a human pathogen causing cutaneous leishmaniasis. This system is based on the expression and (de)stabilization of a basic phospholipase A2 toxin from the Bothrops pauloensis snake venom, which leads to the inducible cell death of the parasites in vitro. Furthermore, the suicidal strain was highly attenuated during macrophage infection, regardless of the toxin stabilization. Such a deliberately weakened parasite could be used to vaccinate the host, as its viability is regulated by the toxin stabilization, causing a profoundly reduced pathogenesis.
\end{abstract}

Keywords: Leishmania mexicana; suicidal system; ecDHFR; BnSP-7; apoptosis

\section{Introduction}

Leishmaniasis is a neglected tropical disease, caused by Leishmania spp., flagellated protozoan parasites belonging to the family Trypanosomatidae [1,2]. The transmission occurs through the bite of a female sand fly (Diptera: Phlebotominae). In the vector, flagellated promastigotes differentiate into the virulent metacyclic forms, which upon injection into the mammalian host develop into the aflagellated, non-motile amastigotes that reside in the phagolysosomes of host macrophages [3,4]. The extent of clinical manifestations of the disease depends both on the parasite species and the immune response developed by the host, but typically ranges from the self-healing cutaneous lesions to fatal visceral infections [1]. Nowadays, leishmaniasis represents the second most serious tropical parasitic disease in terms of mortality and, according to the World Health Organization, over 350 million people live in at-risk endemic areas [5]. Current treatment of the disease is expensive and often associated with severe adverse side effects [6]. Moreover, in many instances a long-lasting parasite resistance develops, underlying the need for an efficient vaccination approach that would allow proper control and prevention of this debilitating disease [7].

To date, several anti-Leishmania vaccination strategies have been proposed. Leishmanization, which is based on the inoculation of low-titer or attenuated live virulent parasites, represents the most efficient approach $[8,9]$. However, its applicability is limited due to apparent safety concerns [10]. Vaccination 
trials based on killed or heat-inactivated parasites, recombinant proteins, and DNA vaccines have shown limitations in murine models and field studies [11]. Another promising experimental approach to this problem is a usage of the genetically modified Leishmania lines as attenuated vaccines [12-16]. There are two aspects that make generation of a successful vaccine particularly challenging. On the one hand, live virulent Leishmania cells are needed to trigger and facilitate complete host immune response, while, on the other hand, such infection must be controllable, so that it does not result in the development of persistent lesions and/or immunosuppression. An inducible expression of toxic genes from suicidal cassettes and subsequent production of suicidal Leishmania strains have been suggested to provide an ideal solution for the aforementioned vaccination limitations: the parasites possess a complete repertoire of virulent components, yet, once the host is immunized, they can be inducibly killed via the intrinsic apoptotic pathways [17-20].

In this study, we introduce an inducible suicidal system in Leishmania mexicana, a causative agent of cutaneous leishmaniasis. A similar system has recently been established in another trypanosomatid species, Trypanosoma cruzi [21]. In both systems, a destabilization domain, derived from the Escherichia coli dihydrofolate reductase (ecDHFR) destabilizes the fusion protein and facilitates its degradation by proteasomes. This process can be reversed by the addition of a specific ligand, Trimethoprim (TMP), or its derivate, Trimethoprim-lactate (TMP-lac) [22]. Screening of a panel of toxins and antimicrobial peptides (AMPs) under axenic conditions revealed that one of them, a basic phospholipase A2 ( $\mathrm{PLA}_{2}$ ) from the Bothrops pauloensis snake venom, called BnSP-7, caused apoptosis-like cell death of the parasites upon the addition of the stabilizing ligand. Noticeably, this toxin has previously been demonstrated to kill L. amazonensis promastigotes in vitro, as well as to delay the amastigote-to-promastigote differentiation, to induce ultrastructural changes in promastigote morphology, and to reduce virulence [23]. The data presented here are complementary to the previous findings. In macrophage infections, the BnSP-7 expressing mutants of L. mexicana showed a significantly reduced infectivity rate compared to its wild type counterparts, even in the absence of the stabilizing agent. Importantly, this attenuated phenotype was further reinforced by the addition of TMP-lac.

We believe that the suicidal system presented here and the resultant of strongly attenuated parasites can be used to develop a novel vaccination strategy against $L$. mexicana, and provide additional insights into the host-derived immune response after elimination of the pathogen. We acknowledge that our findings must be properly reexamined in vivo in animal models of leishmaniasis.

\section{Results}

\subsection{Not All Toxins/Antimicrobial Peptides Are Toxic for Leishmania mexicana Promastigotes}

Growth kinetics was measured for five transgenic L. mexicana cell lines, expressing $\alpha$-toxin, Cecropin-A, Attacin-A, BnSP-7, and Cathelicidin-5, in the presence or absence of the toxin stabilizers, TMP or TMP-lac. We were not able to establish a Leishmania cell line expressing Exotoxin-A, even after multiple attempts. This was probably due to insufficient destabilization and, thus, background expression of the toxin, which resulted in immediate parasite death upon transfection. Of note, all other toxins, fused to ecDHFR-HA, are relatively small compared to the Exotoxin-A, implying that degradation of the toxin-destabilizing domain fusion may be influenced, inter alia, by the toxin size. The growth curves demonstrated that upon addition of the inducer all but one (BnSP-7) toxin-expressing cell lines divided similarly to the wild type Leishmania (Figure S1). The cell morphology was also not affected, supporting the notion that the inducer itself is not detrimental to the parasites. We concluded that $\alpha$-toxin, Cecropin-A, Attacin-A, and Cathelicidin-5 were not toxic for L. mexicana promastigotes.

2.2. BnSP-7, a Basic Phospholipase A2 from Bothrops pauloensis Venom, Dramatically Reduces Viability of L. mexicana Promastigotes In Vitro

The growth of the BnSP-7-ecDHFR-HA-expressing cells was significantly inhibited upon the addition of either TMP or TMP-lac, as compared to the wild type L. mexicana or the untreated 
promastigotes (Figure 1A,B; Table S1). In the absence of induction, the BnSP-7-ecDHFR-HA-expressing cells grow better than the parental ones. When performing an extended growth kinetics analysis, we noticed that the starting concentration of parasites $\left(5 \times 10^{5}\right.$ cells $\left./ \mathrm{mL}\right)$ was decreasing even after 24 hours of TMP treatment (Figure 1C), with no viable cells observed on day 8, whereas the untreated group had reached the stationary log phase on day 6 (Figure 1C). We conclude that BnSP-7 toxin is killing L. mexicana promastigotes in vitro.

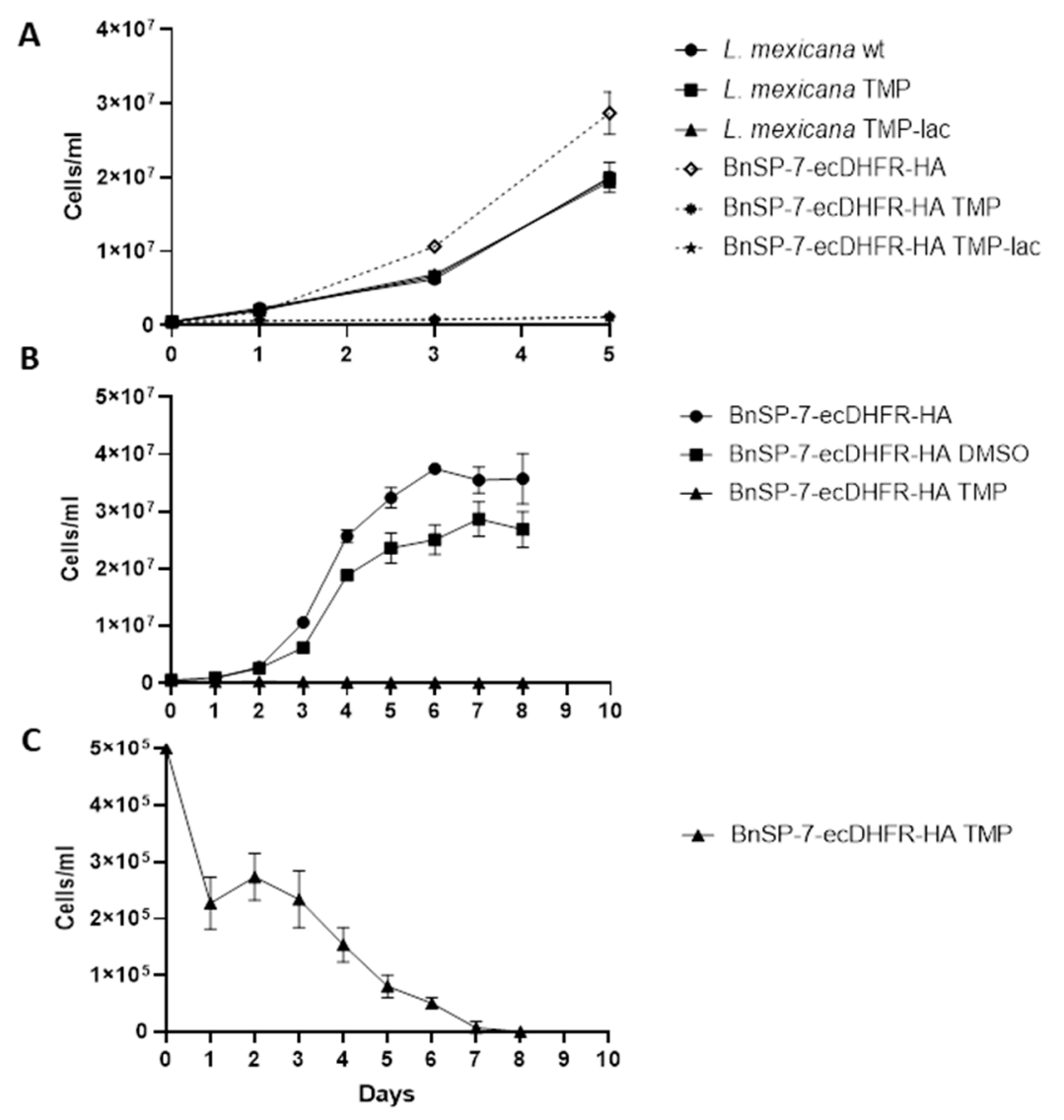

Figure 1. Growth kinetics of Leishmania mexicana expressing the BnSP-7 toxin. (A) Growth curves of the wild type and BnSP-7-ecDHFR-HA-expressing L. mexicana after induction with $20 \mu \mathrm{M}$ Trimethoprim (TMP)/Trimethoprim-lactate (TMP-lac); (B) growth curves of BnSP-7-ecDHFR-HA-expressing L. mexicana, cultivated for 8 days with continuous replenishment of TMP or DMSO; (C) TMP facilitates decrease in a number of BnSP-7-ecDHFR-HA-expressing L. mexicana. All data are derived from three independent biological replicates. The error bars indicate standard deviations.

To confirm this, we checked the early as well as the late apoptotic events by fluorescence microscopy and flow cytometry using the FITC-Annexin V/PI method [24] (Figure 2). As a positive control, the wild type L. mexicana treated with Hygromycin B was used. Cells undergoing apoptosis became abundant in both TMP and TMP-lac-treated conditions. We also noticed that TMP-lac showed slightly lower efficacy, compared to TMP (Figure 2). However, we continued working with TMP-lac due to its solubility in water and, thus, direct applicability for downstream in vivo experiments. 
A
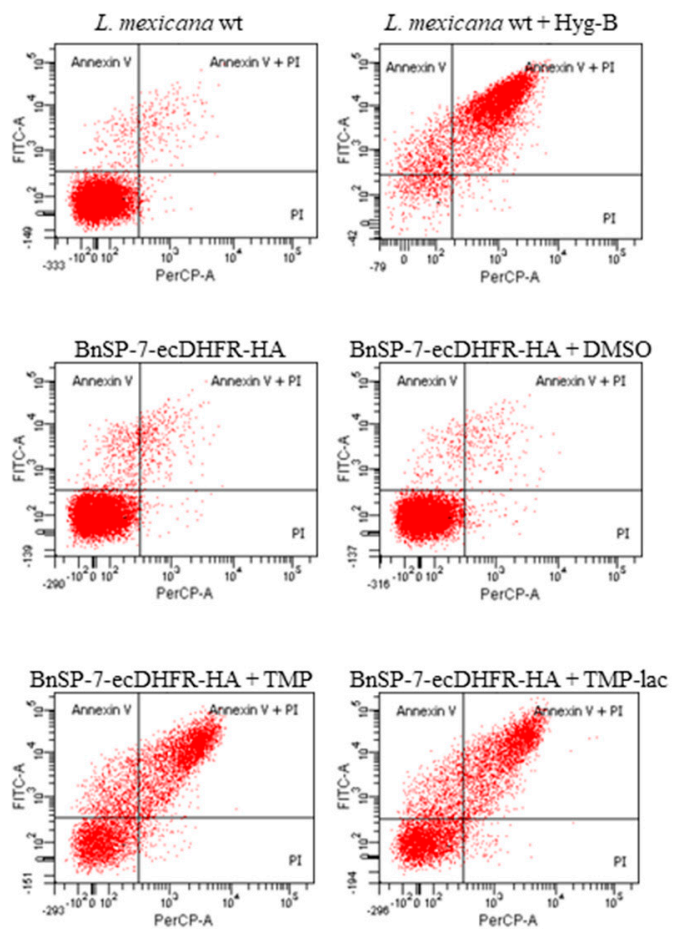

B
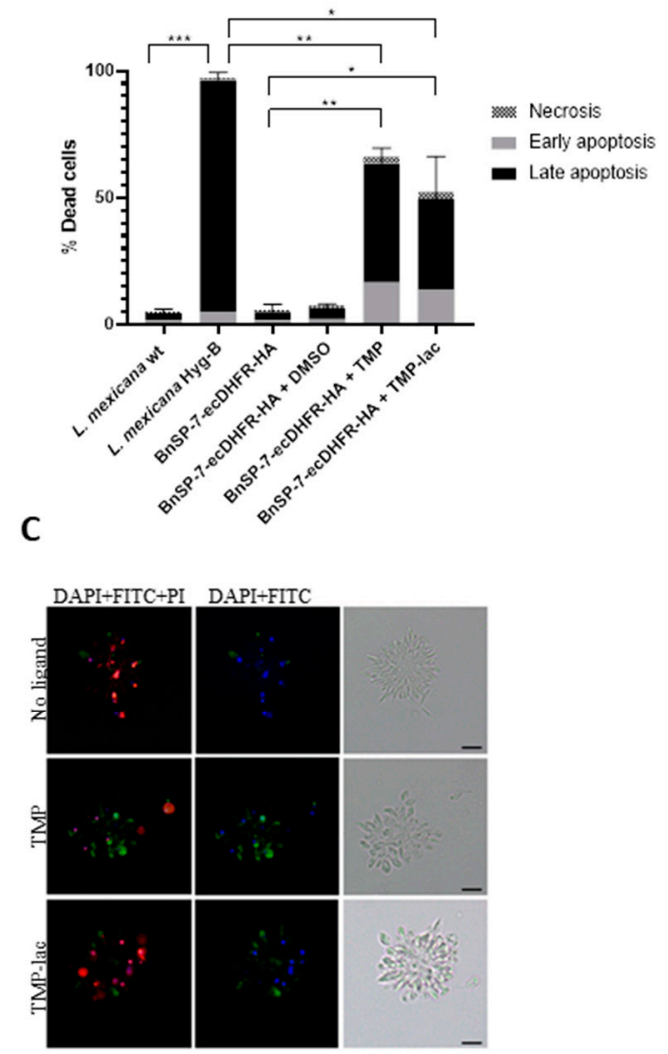

Figure 2. Cell death of L. mexicana expressing the BnSP-7 toxin. (A) Flow cytometry analysis of the L. mexicana cells treated with TMP or TMP-lac for 5 days. Hygromycin B treated wild type L. mexicana cells were used as an apoptotic/necrotic control; (B) apoptosis and necrosis were quantified after FITC-Annexin V and PI labeling; bar graphs represent mean \pm SD in three independent experiments; asterisks indicate $p$ values; ${ }^{*} \leq 0.05,{ }^{* *} \leq 0.01,{ }^{* * *} \leq 0.001$; (C) fluorescence microscopy of the Annexin V-FITC, PI, and DAPI (left panels), Annexin V-FITC and DAPI (middle panels) stained, and light microscopy controls (right panels) of the L. mexicana BnSP-7-ecDHFR-HA cells prior and after induction by the stabilization ligand for 48 hours. Scale bars are $10 \mu \mathrm{m}$.

\subsection{BnSP-7-ecDHFR-HA System Is Functional in Axenically Differentiated L. mexicana Amastigotes}

To check whether stabilization of the toxin protein was not restricted to the promastigote stage, the BnSP-7-ecDHFR-HA-expressing L. mexicana cells were differentiated into amastigotes in vitro and, subsequently, treated with TMP/TMP-lac for 72 hours. Differentiation from the promastigotes into amastigotes in the presence of TMP/TMP-lac was not possible, since the cells died within several days of treatment (Figures 1 and 2). Functionality of the stabilization system was checked by Western blotting, detecting properly processed BnSP-7-HA or BnSp-7- $\mathrm{HA}_{3}$ in both promastigotes (Figure 3A) and cells that were in vitro differentiated into the amastigotes upon the treatment with either TMP or TMP-lac (Figure 3B). The triple HA-tag was introduced for more efficient pull-down. In all cases, protein bands of the expected size, namely $\sim 32 \mathrm{kD}$ for BnSP-7-HA and $\sim 34 \mathrm{kD}$ for BnSp-7-HA 3 , were documented after the TMP/TMP-lac induction. Moreover, the anti-HA antibody also detected a higher $(\sim 40 \mathrm{kD})$ and a lower molecular weight band $(\sim 20 \mathrm{kD})$ in the TMP/TMP-lac-treated parasites. Mass spectrometry analysis showed that these bands represent ubiquitylated and partially degraded forms of BnSP-7-HA or BnSP-7-HA . 
A

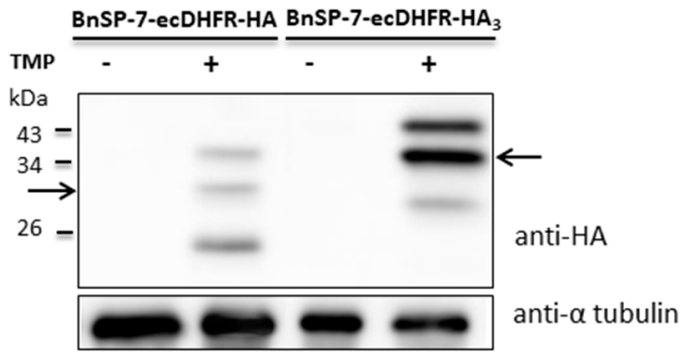

B

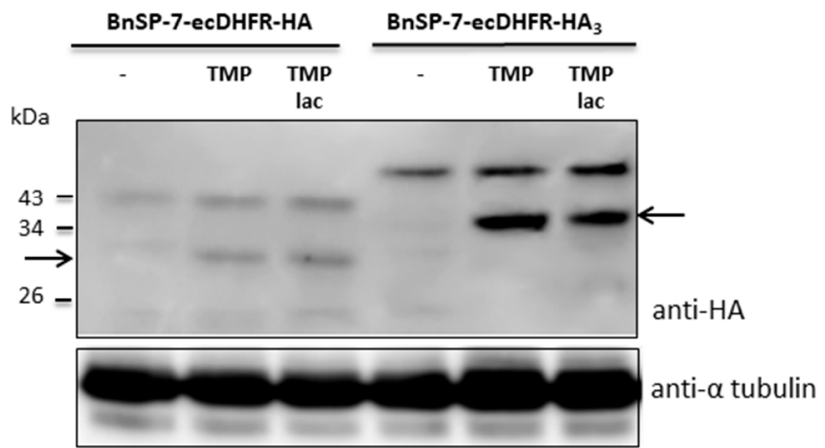

Figure 3. Protein levels of BnSP-7-ecDHFR-HA and BnSP-7-ecDHFR-HA 3 after induction by TMP/TMP-lac in L. mexicana. (A) Western blot of the untreated promastigote lysates and those treated with $20 \mu \mathrm{M}$ TMP for 48 hours; (B) Western blot of the untreated amastigote lysates and those treated with $20 \mu \mathrm{M}$ TMP for 48 hours. Tubulin served as a loading control. Arrows indicate the band of the expected size (32 kDa and $34 \mathrm{kDa}$ for $\mathrm{HA}$ - or $\mathrm{HA}_{3}$-tagged proteins, respectively).

\subsection{The BnSP-7-ecDHFR-HA-expressing L. mexicana Are Attenuated in Macrophage Infections}

Next, J774 macrophages were infected with either the wild type or the BnSP-7-ecDHFR-HAexpressing L. mexicana, followed by the treatment 24 hours post infection (p.i.) with either $20 \mu \mathrm{M}$ TMP-lac or saline buffer, as a control. Four hours p.i., the cells were checked for the proper phagocytic activity and no parasites were observed outside of the macrophages. Importantly, the total number of counted amastigotes (a proxy of Leishmania infectivity) 72 hours p.i. was substantially lower in the case of L. mexicana BnSP-7-ecDHFR-HA as compared to the wild type parasites even without the TMP or TMP-lac treatment (Figure 4A). This implies higher susceptibility of the mutant cells towards macrophage phagocytosis machinery and may reflect the incomplete toxin destabilization inside the macrophages, as the percentage of infected macrophages within the examined groups did not differ (Figure S2). The two-day cultivation of the infected macrophages with $20 \mu \mathrm{M}$ TMP-lac resulted in a $\sim 60 \%$ reduction of the recovered L. mexicana amastigotes, when the number of the BnSP-7-ecDHFR-HA-expressing parasites was compared to their wild type kin (Figure 4B). 
A

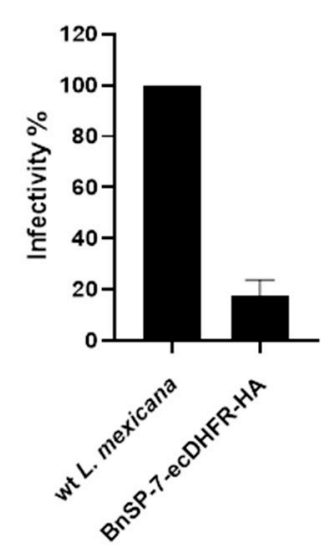

B

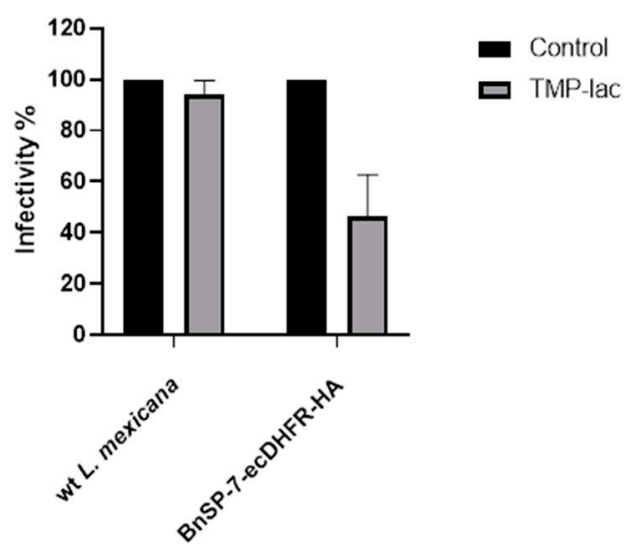

Figure 4. Relative rates of L. mexicana infectivity. (A) Macrophage infection by the wild type and the BnSP-7-ecDHFR-HA-expressing cells; (B) macrophage infection by the wild type and the BnSP-7-ecDHFR-HA-expressing cells treated with $20 \mu \mathrm{M}$ TMP-lac for 48 hours. Data are presented as percentage of the wild type parasites (A) or saline-treated controls (B), taken at $100 \%$. The error bars indicate standard deviation derived from the two independent biological experiments (with 3 technical replicated each).

\section{Discussion}

Toxins and AMPs are natural substances of both prokaryotic and eukaryotic origin [25,26]. Their role is generally related to offensive/defensive responses, resulting in damage or death of the targeted organism $[27,28]$. Some toxins or AMPs are able to kill trypanosomatid parasites, and their trypanocidal and/or leishmanicidal properties have been extensively studied [29]. However, these studies were mostly restricted to the in vitro killing assays with recombinant or synthetic proteins, limiting them primarily to the therapeutic applications.

The aim of this study was to express different toxins/AMPs in L. mexicana using a recently established inducible protein stabilization system [22]. Such genetically-modified suicidal strain of Leishmania would be an eligible and promising candidate for vaccine development. Our search for proteins, whose killing activity was reported either for Trypanosoma or Leishmania spp., resulted in a list of six potential candidates: $\alpha$-toxin, Attacin-A, BnSP-7, Cecropin-A, Cathelicidin-5, and Exotoxin-A. Of note, three of these toxins ( $\alpha$-toxin, Cecropin-A, and Exotoxin-A) have been previously tested in T. cruzi [21].

Insect AMPs Attacin-A, isolated from Glossina morsitans morsitans, and Cecropin-A, obtained from Hyalophora cecropia, fused to the E. coli DHFR degron, did not change the viability of Leishmania mexicana promastigotes upon protein stabilization (Figure S1). They have been previously demonstrated to inhibit growth of T. brucei and T. cruzi [30,31], as well as L. panamensis and L. major amastigotes [32] in vitro. A Bos taurus-derived antimicrobial peptide Cathelicidin-5, whose lethal activity was previously demonstrated for L. major promastigotes [33], was similarly not effective in L. mexicana (Figure S1). AMPs are mostly cationic agents disrupting the membrane integrity by the electrostatic interactions with their anionic phospholipid counterparts [34,35]. Different membrane composition in the Leishmania and Trypanosoma cells (or in different Leishmania spp.) may be responsible for the discrepancy observed for these parasites. This implies that there must be other mechanisms (for example, post-translational modifications, protein stability and folding, etc.), determining whether a toxin will be active against a given parasite species. To illustrate this further, the $\alpha$-toxin from Staphylococcus aureus was the best-choice candidate in T. cruzi studies [21], whereas it had no effect on L. mexicana (Figure S1). This toxin is known to form large $\mathrm{Ca}^{2+}$-permissive pores in the cellular membrane, resulting in a massive homeostasis disruption and cell death [36]. Because the $\alpha$-toxin forms a heptameric transmembrane pore [37], we presumed that its fusion with a destabilizing domain has interfered with its proper folding. In contrast, all our attempts to generate a cell line, expressing Pseudomonas aeruginosa 
Exotoxin-A, have invariably failed. We explained this by insufficient toxin destabilization, followed by the inhibition of protein synthesis and cell death. Overall, the data presented here indicate that the functionality of a suicidal system relies both on the selected toxin and the host used for its expression.

The BnSP-7, a catalytically inactive Phospholipase A2 ( $\left.\mathrm{PLA}_{2}\right)$ homolog from the Bothrops pauloensis snake venom, was the only, albeit very effective, toxin, acting against $L$. mexicana promastigotes and amastigotes, identified in this study. Although lacking the catalytic activity due to a single amino acid substitution (Asp49Lys), myotoxic PLA2 homologs have the ability to disrupt biological membranes in a $\mathrm{Ca}^{2+}$-independent manner not involving the hydrolysis of phospholipids [38-40]. In cytotoxicity assays, its activity has also been demonstrated against several Leishmania spp. [41-43]. The pilot TMP/TMP-lac treatment experiments with the BnSP-7-ecDHFR-HA-expressing parasites have confirmed the anti-leishmanial potential of this toxin (Figure 1). Cells did not multiply and their morphology dramatically changed from long promastigotes to round-shaped cells, indicating apoptosis. Moreover, the number of parasites was decreasing after 72 hours of continuous TMP treatment, reaching an undetectable level by day 8 (Figure 1C). Flow cytometry and fluorescence microscopy evaluation $[44,45]$ confirmed the apoptotic nature of the toxin-induced Leishmania cell death (Figure 2).

Amastigotes could not be differentiated in the presence of BnSP-7. To overcome this, we first produced amastigotes in vitro and then treated them with either TMP or TMP-lac. The readout of this experiment was a stabilized toxin, detected by immunoblotting. Both the promastigotes and the amastigotes showed the same expression pattern with three bands upon addition of the stabilizing agent (Figure 3). Immunoprecipitation followed by mass spectrometry analysis confirmed the identity of BnSP-7-ecDHFR-HA, its degradation product, and the post-translationally modified mono-ubiquitylated form. In both promastigotes and amastigotes, the functional toxin was predominant after stabilization. Recent studies have shown that mono-ubiquitylation of smaller proteins can target them for proteasomal recognition and processing [46]. We assume that mono-ubiquitylation of the fused destabilized protein drives its proteolysis, and the domain stabilization by TMP/TMP-lac interferes with this process.

When performing the macrophage infections, we noticed a surprisingly reduced infectivity of the BnSP-7-ecDHFR-HA-expressing L. mexicana even in the absence of the TMP-facilitated stabilization (Figure 4A). Since the phagocytic activity of macrophages did not differ between the wild type L. mexicana and the mutant, we presume that the BnSP-7-ecDHFR-HA-expressing parasites possess all antigens required for macrophage recognition, but their viability and/or division rate was lowered after internalization into the phagolysosomes. The most parsimonious explanation of such an attenuated phenotype is that the toxin was partly stabilized in the phagolysosomal environment, thus resulting in a reduced infectivity. As expected, the TMP-lac treatment further decreased the number of recovered amastigotes in the case of the mutant cell line compared to the wild type L. mexicana (Figure 4B). The BnSP-7 toxin expression causes elevated susceptibility upon macrophage engulfment and this can be enhanced via an inducible (de)stabilization system. We realize that such a highly attenuated mutant strain of L. mexicana needs to be reexamined in vivo in animal models of leishmaniasis, as the immune response is too complex to be reliably predicted from the in vitro studies only.

In conclusion, this is the first report of the basic PLA 2 homologue, derived from the Bothrops pauloensis snake venom, with lethal activity against L. mexicana. We believe that these results may ultimately lead to the development of novel vaccination strategies based on the controllably suicidal Leishmania.

\section{Materials and Methods}

\subsection{Axenic Cultivation of Leishmania mexicana}

Leishmania mexicana (MNYC/BZ/1962/M379) promastigotes were cultured at $23{ }^{\circ} \mathrm{C}$ in the M199 medium (Sigma-Aldrich, Saint. Louis, MO, USA), supplemented with $2 \mu \mathrm{g} / \mathrm{mL}$ Biopterin 
(Sigma-Aldrich), 2 g/mL Hemin (Jena Bioscience GmbH, Jena, Germany), 25 mM HEPES, 50 units/mL of penicillin/streptomycin (Life Technologies, Carlsbad, CA, USA), and 10\% Fetal Bovine Serum (FBS, BioSera Europe, Nuaillé, France). The amastigotes of L. mexicana were differentiated in vitro and cultivated at $32{ }^{\circ} \mathrm{C}$ in the complete M199 medium (pH 5.5), supplemented with 20\% FBS [47,48]. The following ligands were tested: Trimethoprim (Sigma-Aldrich, $10 \mathrm{mM}$ stock in DMSO) and Trimethoprim-lactate (TMP-lac, Sigma-Aldrich, $10 \mathrm{mM}$ stock in M199, or $1 \mathrm{mM}$ stock in a saline buffer).

\subsection{Genetic Manipulations}

Information on the toxins and AMPs analyzed in this study is summarized in Table 1. The open reading frames (ORFs) of $\alpha$-toxin, Cecropin-A, and Exotoxin-A were PCR amplified from the pTREX-based constructs (kindly provided by Dr. Huang [21]) using primers A1/A2, C1/C2, and E1/E2, respectively (Table S2). These fragments were fused with the modified ecDHFR-HA domain (mut2 in [22]), amplified from the pLEXSY-based construct with primers D1/D2, using nested primers A3, C3, E3, and D3 (Table S2) as described previously [49]. The fragments encoding ecDHFR-HA (mut2) fusions of Attacin-A, BnSP-7, and Cathelicidin-5 (GenBank accession numbers AY607104, AF145781, and X97609, respectively), were synthesized de novo by GeneCust Europe (Boynes, France). All fragments were cloned into the pLEXSY-hyg2 (Jena Bioscience $\mathrm{GmbH}$ ) using restriction enzymes $B g l \mathrm{II}$ (Thermo Fisher Scientific, Waltham, MA, USA) and NotI (New England Biolabs, Ipswich, MA, USA). All resulting plasmids were linearized with SwaI (New England Biolabs), gel purified, and transfected into the log phase-grown procyclic promastigotes of L. mexicana using BTX ECM 630 electroporator (Harvard Apparatus Inc, Holliston, MA, USA), as described previously [50]. The transfectants were selected for 10 days in the cultivation medium, supplemented with $200 \mu \mathrm{g} / \mathrm{mL}$ of Hygromycin B (Carl Roth GmbH, Karlsruhe, Germany).

Table 1. List of toxins/antimicrobial peptides fused with mut 2 ecDHFR-HA [22] and their toxic properties.

\begin{tabular}{cccc}
\hline AMP/Toxin & Source & Mass (kDa) & Activity \\
\hline Alpha-toxin & Staphylococcus aureus & 35.9 & plasma membrane permeabilization \\
Attacin-A & Glossina morsitans morsitans & 21.7 & plasma membrane permeabilization \\
BnSP-7 & Bothrops pauloensis & 13.7 & plasma membrane permeabilization \\
Cathelicidin-5 & Bos taurus & 17.6 & plasma membrane permeabilization \\
(BMAP-28) & Hyalophora cecropia & 6.9 & plasma membrane permeabilization \\
Cecropin-A & Pseudomonas aeruginosa & 69 & elongation factor-2 inhibition \\
Exotoxin-A & & &
\end{tabular}

In addition, the BnSP-7-ecDHFR-HA fragment was re-amplified from the pLEXSY construct using primers $\mathrm{B} 1$ and D4. The resulting $\mathrm{HA}_{3}$-tagged construct was cloned back into the pLEXSY-hyg2 plasmid and transfected as described above. PCR confirmation of the correct integration was performed using primers SSU_F and HA_R (Table S2).

\subsection{Growth Kinetics and Differentiation}

Growth kinetics analysis of the wild type and transgenic cell lines was performed for 5 days from the starting density of $5 \times 10^{5}$ cells/mL in three biological replicates, as described previously [51]. The counting was also performed for all transfectants after the addition of $20 \mu \mathrm{M}$ TMP or TMP-lac on day 0 , using wild type L. mexicana as a control. The BnSP-7-ecDHFR-HA expressing L. mexicana cells were additionally counted in the same manner every 24 hours for 8 days with daily replenishment of TMP.

Differentiation of the BnSP-7-ecDHFR-HA expressing L. mexicana mutants into amastigotes was performed by varying $\mathrm{pH}$ and temperature as described previously [48]. Once the parasites reached 
the amastigote stage (day 17), they were treated with $20 \mu \mathrm{M}$ TMP/TMP-lac and analyzed by Western blotting after 48 hours.

\subsection{Apoptosis Assays}

The analysis of L. mexicana apoptosis was carried out using the eBioscience Annexin V Apoptosis Detection Kit FITC (Thermo Fisher Scientific) according to the manufacturer's instructions. Briefly, $5 \times 10^{6} / \mathrm{mL}$ of the BnSP-7-ecDHFR-HA expressing promastigotes, incubated with or without $20 \mu \mathrm{M}$ TMP, TMP-lac, or DMSO, were harvested, washed, and treated with $5 \mu \mathrm{L}$ of FITC-Annexin V for 15 min at room temperature in the dark, followed by treatment with $5 \mu \mathrm{L}$ propidium iodide (PI, $20 \mu \mathrm{g} / \mathrm{mL}$ ). The cells were then analyzed on a FACSCanto II flow cytometer (Becton Dickinson, Franklin Lakes, NJ, USA) or visualized directly by fluorescent microscopy using the Olympus BX51 microscope equipped with a DP70 charge-coupled device camera (Olympus, Tokyo, Japan). As a positive control, L. mexicana wild type cells, treated by Hygromycin B, were used. Data were collected from three independent biological replicates and tested for the significance using two-tailed, paired Student $t$-test. $p$ values $\leq 0.05$ were considered statistically significant.

\subsection{Immunoblotting, Immunoprecipitation, and Mass Spectrometry Analysis}

All used antibodies were from Sigma-Aldrich. Protein lysates (10 $\mu$ g per lane) were separated in 10\% SDS-PAGE, transferred and treated with polyclonal rabbit anti-HA $(1: 1,000)$ and monoclonal mouse anti- $\alpha$-tubulin $(1: 2,000)$ primary antibodies, combined with HRP-labeled goat anti-rabbit IgG $(1: 80,000)$ and rabbit anti-mouse IgG $(1: 80,000)$ secondary antibodies, respectively.

The immunoprecipitation of triple HA-tagged BnSP-7-ecDHFR was carried out with the L. mexicana protein lysates using Pierce Anti-HA magnetic beads (Thermo Fisher Scientific), following the manufacturer's instructions. Elution of the sample was performed by heat denaturation in Laemmli sample buffer (Bio-Rad, Hercules, CA, USA) for 10 minutes. Samples were separated in 10\% SDS-PAGE gels, stained with Coomassie Brilliant Blue G-250 (Thermo Fisher Scientific), cut out and sent for liquid chromatography-tandem mass spectrometry (LC-MS/MS) analysis (CEITEC, Brno, Czech Republic).

\subsection{Macrophage Infection}

The L. mexicana wild type promastigotes were cultured in the M199 medium (Sigma-Aldrich) containing 10\% FBS supplemented with 1\% Basal Medium Eagle vitamins (Sigma-Aldrich), 2\% sterile urine, and $250 \mu \mathrm{g} / \mathrm{mL}$ Amikacin (Bristol-Myers Squibb, New York, NY, USA), and $200 \mu \mathrm{g} / \mathrm{mL}$ of Hygromycin B (Carl Roth $\mathrm{GmbH}$ ) for BnSP-7-ecDHFR-HA mutant cell lines. Infection of the J774 macrophages was performed as described previously $[49,52]$. In addition, infection was evaluated by counting Giemsa-stained slides as described previously [51]. All experiments were performed with two independent biological replicates and samples were analyzed in triplicate.

Supplementary Materials: The following are available online at http://www.mdpi.com/2076-0817/9/2/79/s1, Figure S1: Growth curves of Leishmania mexicana encoding different toxins fused to ecDHFR destabilizing domain (mut 2 in [22]), with and without induction by stabilizing ligands, TMP and TMP-lac $(20 \mu \mathrm{M})$. Red, wild type L. mexicana; violet, Attacin-A-ecDHFR-HA expressing L. mexicana; blue, Alpha-toxin-ecDHFR-HA expressing L. mexicana; neon green, BnSP-7-ecDHFR-HA expressing L. mexicana; brown, Cathelicidin-5-ecDHFR-HA expressing L. mexicana; green, Cecropin-A ecDHFR-HA expressing L. mexicana, Figure S2: Percentage of infected macrophages by the wild type and BnSP-7-ecDHFR-HA cell line non-treated (control) and treated with TMP-lac, Table S1: Growth kinetics of the wild type and BnSP-7-ecDHFR-HA-expressing L. mexicana after induction with $20 \mu \mathrm{M}$ TMP/TMP-lac; standard deviations are indicated in the brackets, Table S2: List of primers used in this study.

Author Contributions: Conceptualization, V.Y.; methodology, V.Y. and L.P.; formal analysis, L.P.; investigation, L.P., T.L., and E.H.; resources, V.Y., J.L., and P.V.; data curation, L.P.; writing-original draft preparation, L.P.; writing-review and editing, L.P., T.L., E.H., J.L., P.V., and V.Y.; supervision, V.Y., J.L., and P.V; funding acquisition, V.Y., T.L., J.L., and P.V. All authors have read and agreed to the published version of the manuscript. 
Funding: This research was funded by the Russian Science Foundation (grant 19-15-00054 to V.Y., concept of suicidal Leishmania, in vitro experiments); European Research Council CZ LL1601 (to J.L.); European Regional Funds (project "Centre for Research of Pathogenicity and Virulence of Parasites" CZ.02.1.01/0.0/ 0.0/16_019/0000759 to V.Y., J.L., P.V., and L.P.); Grant Agency of Charles University (UNCE 20472 to T.L.); and a grant from the University of Ostrava (to V.Y.). We acknowledge the infrastructure grant "Přístroje IET" (CZ.1.05/2.1.00/19.0388).

Acknowledgments: We thank Huang (Albert Einstein College of Medicine, Bronx, NY, USA) for providing the plasmids and the members of our laboratories for the stimulating discussions.

Conflicts of Interest: V.Y. is an Academic Editor of Pathogens. Other authors declare no conflict of interest. The funders had no role in the design of the study; in the collection, analyses, or interpretation of data; in the writing of the manuscript, or in the decision to publish the results.

\section{References}

1. Bruschi, F.; Gradoni, L. The Leishmaniases: Old Neglected Tropical Diseases; Springer: Cham, Switzerland, 2018; p. 245.

2. Maslov, D.A.; Opperdoes, F.R.; Kostygov, A.Y.; Hashimi, H.; Lukeš, J.; Yurchenko, V. Recent advances in trypanosomatid research: Genome organization, expression, metabolism, taxonomy and evolution. Parasitology 2019, 146, 1-27. [CrossRef]

3. Dostálová, A.; Volf, P. Leishmania development in sand flies: Parasite-vector interactions overview. Parasites Vectors 2012, 5, 276. [CrossRef] [PubMed]

4. Podinovskaia, M.; Descoteaux, A. Leishmania and the macrophage: A multifaceted interaction. Future Microbiol. 2015, 10, 111-129. [CrossRef] [PubMed]

5. WHO. Leishmaniasis: Stuation and Trends. Global Health Observatory (GHO) Data 2016. Available online: http://www.who.int/gho/neglected_diseases/leishmaniasis/en/ (accessed on 24 January 2020).

6. Oliveira, L.F.; Schubach, A.O.; Martins, M.M.; Passos, S.L.; Oliveira, R.V.; Marzochi, M.C.; Andrade, C.A. Systematic review of the adverse effects of cutaneous leishmaniasis treatment in the New World. Acta Trop. 2011, 118, 87-96. [CrossRef] [PubMed]

7. Rezvan, H.; Moafi, M. An overview on Leishmania vaccines: A narrative review article. In Veterinary Research Forum; Faculty of Veterinary Medicine, Urmia University: Urmia, Iran, 2015; Volume 6, pp. 1-7.

8. McCall, L.I.; Zhang, W.W.; Ranasinghe, S.; Matlashewski, G. Leishmanization revisited: Immunization with a naturally attenuated cutaneous Leishmania donovani isolate from Sri Lanka protects against visceral leishmaniasis. Vaccine 2013, 31, 1420-1425. [CrossRef]

9. Khamesipour, A.; Dowlati, Y.; Asilian, A.; Hashemi-Fesharki, R.; Javadi, A.; Noazin, S.; Modabber, F. Leishmanization: Use of an old method for evaluation of candidate vaccines against leishmaniasis. Vaccine 2005, 23, 3642-3648. [CrossRef]

10. Handman, E. Leishmaniasis: Current status of vaccine development. Clin. Microbiol. Rev. 2001, 14, $229-243$. [CrossRef]

11. Kedzierski, L. Leishmaniasis vaccine: Where are we today? J. Glob. Infect. Dis. 2010, 2, 177-185. [CrossRef]

12. Santi, A.M.M.; Lanza, J.S.; Tunes, L.G.; Fiuza, J.A.; Roy, G.; Orfano, A.D.S.; de Carvalho, A.T.; Frezard, F.; Barros, A.L.B.; Murta, S.M.F.; et al. Growth arrested live-attenuated Leishmania infantum KHARON1 null mutants display cytokinesis defect and protective immunity in mice. Sci. Rep. 2018, 8, 11627. [CrossRef]

13. Dey, R.; Dagur, P.K.; Selvapandiyan, A.; McCoy, J.P.; Salotra, P.; Duncan, R.; Nakhasi, H.L. Live attenuated Leishmania donovani 27 gene knockout parasites are nonpathogenic and elicit long-term protective immunity in BALB/c mice. J. Immunol. 2013, 190, 2138-2149. [CrossRef]

14. Selvapandiyan, A.; Dey, R.; Nylen, S.; Duncan, R.; Sacks, D.; Nakhasi, H.L. Intracellular replication-deficient Leishmania donovani induces long lasting protective immunity against visceral leishmaniasis. J. Immunol. 2009, 183, 1813-1820. [CrossRef] [PubMed]

15. Anand, S.; Madhubala, R. Genetically engineered ascorbic acid-deficient live mutants of Leishmania donovani induce long lasting protective immunity against visceral leishmaniasis. Sci. Rep. 2015, 5, 10706. [CrossRef]

16. Solana, J.C.; Ramirez, L.; Corvo, L.; de Oliveira, C.I.; Barral-Netto, M.; Requena, J.M.; Iborra, S.; Soto, M. Vaccination with a Leishmania infantum HSP70-II null mutant confers long-term protective immunity against Leishmania major infection in two mice models. PLoS Negl. Trop. Dis. 2017, 11, e0005644. [CrossRef] [PubMed]

17. Muyombwe, A.; Olivier, M.; Ouellette, M.; Papadopoulou, B. Selective killing of Leishmania amastigotes expressing a thymidine kinase suicide gene. Exp. Parasitol. 1997, 85, 35-42. [CrossRef] [PubMed] 
18. Ghedin, E.; Charest, H.; Zhang, W.W.; Debrabant, A.; Dwyer, D.; Matlashewski, G. Inducible expression of suicide genes in Leishmania donovani amastigotes. J. Biol. Chem. 1998, 273, 22997-23003. [CrossRef] [PubMed]

19. Davoudi, N.; Tate, C.A.; Warburton, C.; Murray, A.; Mahboudi, F.; McMaster, W.R. Development of a recombinant Leishmania major strain sensitive to ganciclovir and 5-fluorocytosine for use as a live vaccine challenge in clinical trials. Vaccine 2005, 23, 1170-1177. [CrossRef]

20. Kumari, S.; Samant, M.; Khare, P.; Misra, P.; Dutta, S.; Kolli, B.K.; Sharma, S.; Chang, K.P.; Dube, A. Photodynamic vaccination of hamsters with inducible suicidal mutants of Leishmania amazonensis elicits immunity against visceral leishmaniasis. Eur. J. Immunol. 2009, 39, 178-191. [CrossRef]

21. Ma, Y.; Weiss, L.M.; Huang, H. Inducible suicide vector systems for Trypanosoma cruzi. Microbes Infect. 2015, 17, 440-450. [CrossRef]

22. Podesvova, L.; Huang, H.; Yurchenko, V. Inducible protein stabilization system in Leishmania mexicana. Mol. Biochem. Parasitol. 2017, 214, 62-64. [CrossRef]

23. Nunes, D.C.; Figueira, M.M.; Lopes, D.S.; de Souza, D.L.; Izidoro, L.F.; Ferro, E.A.; Souza, M.A.; Rodrigues, R.S.; Rodrigues, V.M.; Yoneyama, K.A. BnSP-7 toxin, a basic phospholipase A2 from Bothrops pauloensis snake venom, interferes with proliferation, ultrastructure and infectivity of Leishmania (Leishmania) amazonensis. Parasitology 2013, 140, 844-854. [CrossRef]

24. Vermes, I.; Haanen, C.; Steffens-Nakken, H.; Reutelingsperger, C. A novel assay for apoptosis. Flow cytometric detection of phosphatidylserine expression on early apoptotic cells using fluorescein labelled Annexin V. J. Immunol. Methods 1995, 184, 39-51. [CrossRef]

25. Schlamadinger, D.E.; Gable, J.E.; Kim, J.E. Toxins and antimicrobial peptides: Interactions with membranes. Proc. SPIE Int. Soc. Opt. Eng. 2009, 7397, 73970J. [PubMed]

26. Makarova, K.S.; Wolf, Y.I.; Karamycheva, S.; Zhang, D.; Aravind, L.; Koonin, E.V. Antimicrobial peptides, polymorphic toxins, and self-nonself recognition systems in Archaea: An untapped armory for intermicrobial conflicts. MBio 2019, 10, e00715-19. [CrossRef] [PubMed]

27. Lukša, J.; Ravoitytè, B.; Konovalovas, A.; Aitmanaitè, L.; Butenko, A.; Yurchenko, V.; Serva, S.; Servienè, E. Different metabolic pathways are involved in response of Saccharomyces cerevisiae to L-A and M viruses. Toxins 2017, 9, e233. [CrossRef] [PubMed]

28. Harms, A.; Brodersen, D.E.; Mitarai, N.; Gerdes, K. Toxins, targets, and triggers: An overview of toxin-antitoxin biology. Mol. Cell 2018, 70, 768-784. [CrossRef]

29. Müller, S.; Cerdan, R.; Radulescu, O. Drug discovery in infectious diseases. In Comprehensive Analysis of Parasite Biology: From Metabolism to Drug Discovery; Selzer, P.M., Ed.; Wiley-VCH: Weinheim, Germany, 2016; Volume 7, p. 549.

30. Hu, Y.; Aksoy, S. An antimicrobial peptide with trypanocidal activity characterized from Glossina morsitans morsitans. Insect Biochem. Mol. Biol. 2005, 35, 105-115. [CrossRef] [PubMed]

31. Fieck, A.; Hurwitz, I.; Kang, A.S.; Durvasula, R. Trypanosoma cruzi: Synergistic cytotoxicity of multiple amphipathic anti-microbial peptides to T. cruzi and potential bacterial hosts. Exp. Parasitol. 2010, 125, 342-347. [CrossRef]

32. Pérez-Cordero, J.J.; Lozano, J.M.; Cortes, J.; Delgado, G. Leishmanicidal activity of synthetic antimicrobial peptides in an infection model with human dendritic cells. Peptides 2011, 32, 683-690. [CrossRef]

33. Lynn, M.A.; Kindrachuk, J.; Marr, A.K.; Jenssen, H.; Pante, N.; Elliott, M.R.; Napper, S.; Hancock, R.E.; McMaster, W.R. Effect of BMAP-28 antimicrobial peptides on Leishmania major promastigote and amastigote growth: Role of leishmanolysin in parasite survival. PLoS Negl. Trop. Dis. 2011, 5, e1141. [CrossRef]

34. Epand, R.M.; Vogel, H.J. Diversity of antimicrobial peptides and their mechanisms of action. Biochim. Biophys. Acta 1999, 1462, 11-28. [CrossRef]

35. Jenssen, H.; Hamill, P.; Hancock, R.E. Peptide antimicrobial agents. Clin. Microbiol. Rev. 2006, 19, 491-511. [CrossRef]

36. Bantel, H.; Sinha, B.; Domschke, W.; Peters, G.; Schulze-Osthoff, K.; Janicke, R.U. alpha-Toxin is a mediator of Staphylococcus aureus-induced cell death and activates caspases via the intrinsic death pathway independently of death receptor signaling. J. Cell Biol. 2001, 155, 637-648. [CrossRef]

37. Song, L.; Hobaugh, M.R.; Shustak, C.; Cheley, S.; Bayley, H.; Gouaux, J.E. Structure of staphylococcal alpha-hemolysin, a heptameric transmembrane pore. Science 1996, 274, 1859-1866. [CrossRef] [PubMed] 
38. Lomonte, B.; Angulo, Y.; Sasa, M.; Gutierrez, J.M. The phospholipase A2 homologues of snake venoms: Biological activities and their possible adaptive roles. Protein Pept. Lett. 2009, 16, 860-876. [CrossRef] [PubMed]

39. Gutiérrez, J.M.; Lomonte, B. Phospholipase A2 myotoxins from Bothrops snake venoms. Toxicon 1995, 33, 1405-1424. [CrossRef]

40. Núñez, C.E.; Angulo, Y.; Lomonte, B. Identification of the myotoxic site of the Lys49 phospholipase A(2) from Agkistrodon piscivorus piscivorus snake venom: Synthetic C-terminal peptides from Lys49, but not from Asp49 myotoxins, exert membrane-damaging activities. Toxicon 2001, 39, 1587-1594. [CrossRef]

41. Grabner, A.N.; Alfonso, J.; Kayano, A.M.; Moreira-Dill, L.S.; Santos, A.P.A.D.; Caldeira, C.A.S.; Sobrinho, J.C.; Gomez, A.; Grabner, F.P.; Cardoso, F.F.; et al. BmajPLA2-II, a basic Lys49-phospholipase A2 homologue from Bothrops marajoensis snake venom with parasiticidal potential. Int. J. Biol. Macromol. 2017, 102, 571-581. [CrossRef]

42. Passero, L.F.; Laurenti, M.D.; Tomokane, T.Y.; Corbett, C.E.; Toyama, M.H. The effect of phospholipase A2 from Crotalus durissus collilineatus on Leishmania (Leishmania) amazonensis infection. Parasitol. Res. 2008, 102, 1025-1033. [CrossRef]

43. Alfonso, J.J.; Kayanoa, A.M.; Garay, A.F.G.; Simoes-Silva, R.; Sobrinho, J.C.; Vourliotis, S.; Soares, A.M.; Calderon, L.A.; Gomez, M.C.V. Isolation, biochemical characterization and antiparasitic activity of BmatTX-IV, a basic Lys49-phospholipase A2 from the venom of Bothrops mattogrossensis from Paraguay. Curr. Top. Med. Chem. 2019, 19, 2041-2048. [CrossRef]

44. Basmaciyan, L.; Berry, L.; Gros, J.; Azas, N.; Casanova, M. Temporal analysis of the autophagic and apoptotic phenotypes in Leishmania parasites. Microb. Cell 2018, 5, 404-417. [CrossRef]

45. Weingärtner, A.; Kemmer, G.; Muller, F.D.; Zampieri, R.A.; dos Santos, M.G.; Schiller, J.; Pomorski, T.G. Leishmania promastigotes lack phosphatidylserine but bind annexin $\mathrm{V}$ upon permeabilization or miltefosine treatment. PLoS ONE 2012, 7, e42070. [CrossRef] [PubMed]

46. Braten, O.; Livneh, I.; Ziv, T.; Admon, A.; Kehat, I.; Caspi, L.H.; Gonen, H.; Bercovich, B.; Godzik, A.; Jahandideh, S.; et al. Numerous proteins with unique characteristics are degraded by the $26 \mathrm{~S}$ proteasome following monoubiquitination. Proc. Natl. Acad. Sci. USA 2016, 113, E4639-E4647. [CrossRef] [PubMed]

47. Bates, P.A.; Tetley, L. Leishmania mexicana: Induction of metacyclogenesis by cultivation of promastigotes at acidic pH. Exp. Parasitol. 1993, 76, 412-423. [CrossRef] [PubMed]

48. Bates, P.A. Axenic culture of Leishmania amastigotes. Parasitol. Today 1993, 9, 143-146. [CrossRef]

49. Ishemgulova, A.; Kraeva, N.; Hlavacova, J.; Zimmer, S.L.; Butenko, A.; Podesvova, L.; Lestinova, T.; Lukes, J.; Kostygov, A.; Votypka, J.; et al. A putative ATP/GTP binding protein affects Leishmania mexicana growth in insect vectors and vertebrate hosts. PLoS Negl. Trop. Dis. 2017, 11, e0005782. [CrossRef]

50. Kraeva, N.; Ishemgulova, A.; Lukeš, J.; Yurchenko, V. Tetracycline-inducible gene expression system in Leishmania mexicana. Mol. Biochem. Parasitol. 2014, 198, 11-13. [CrossRef]

51. Kraeva, N.; Butenko, A.; Hlaváčová, J.; Kostygov, A.; Myškova, J.; Grybchuk, D.; Leštinová, T.; Votýpka, J.; Volf, P.; Opperdoes, F.; et al. Leptomonas seymouri: Adaptations to the dixenous life cycle analyzed by genome sequencing, transcriptome profiling and co-infection with Leishmania donovani. PLoS Pathog. 2015, 11, e1005127. [CrossRef]

52. Kraeva, N.; Leštinová, T.; Ishemgulova, A.; Majerová, K.; Butenko, A.; Vaselek, S.; Bespyatykh, J.; Charyyeva, A.; Spitzová, T.; Kostygov, A.Y.; et al. LmxM.22.0250-encoded dual specificity protein/lipid phosphatase impairs Leishmania mexicana virulence in vitro. Pathogens 2019, 8, 241. [CrossRef]

(C) 2020 by the authors. Licensee MDPI, Basel, Switzerland. This article is an open access article distributed under the terms and conditions of the Creative Commons Attribution (CC BY) license (http://creativecommons.org/licenses/by/4.0/). 